\title{
BUCKLING IMPERFECTION SENSITIVITY OF COLUMNS AND SPHERICAL CAPS*
}

$\mathrm{Bx}$

\author{
J. P. KEENER
}

University of Arizona

Introduction. Imperfection-sensitivity and post-buckling theory have been the subject of extensive studies in recent years [4], [6]. In these studies (see for example [2], [7]) the critical buckling load is defined to be the maximum load attainable on the pre-buckling portion of the load-displacement curve of an imperfect structure. This definition leads to various approximations of the load-displacement curve, from which the buckling load can then be estimated.

In this paper we present a very general iteration procedure for calculating the buckling load of imperfect structures. The method does not require very special knowledge about the form of the imperfections, and the iterates can be rigorously shown to converge for small imperfections and to give asymptotically valid estimates of the buckling load as a function of the imperfection amplitude. The method employs an alternate definition of the buckling load which for imperfection-sensitive structures gives the same buckling load as the more standard approach. The method is also valid for problems which are imperfection-"insensitive," even though the prebuckling curve does not have a maximum attainable load. In this case, the method determines the imperfection sensitivity of certain "unbuckling" loads. For a treatment of a problem of this type, see [10].

In the following sections we show how these techniques are easily applied to two specific problems. In Sec. I we calculate the buckling load of an imperfect column on a nonlinearly elastic foundation. In Section II we discuss the buckling of a spherical cap under a number of conditions. Convergence proofs and other theoretical aspects of the method are given elsewhere [8], [9].

I. Buckling of a uniform column on a nonlinearly elastic foundation. Consider a pinned uniform column with an initial imperfection resting on a "softening" nonlinear elastic foundation and subjected to an axial load. For small deflections, the governing nondimensional equation is taken to be [2]

$\frac{d^{4} w}{d x^{4}}+2 \lambda \frac{d^{2} w}{d x^{2}}+w-w^{3}=-2 \lambda \tau \frac{d^{2} w_{0}}{d x^{2}}$

$$
w(0)=w(\pi)=0, \quad w^{\prime \prime}(0)=w^{\prime \prime}(\pi)=0,
$$

where $w(x)$ represents the lateral displacement and $\lambda$ represents the axial loading, with

* Received July 3, 1972; revised version received January 20, 1973. This research was done while the author held a fellowship from the Fannie and John Hertz Foundation. 
$\lambda>0$ for compressive loads. The initial imperfection has shape $w_{0}(x)$ and amplitude $\tau$. This problem has been considered in [2] for columns of infinite length and for some special imperfection shapes $w_{0}(x)$. The treatment given here requires only very modest assumptions on $w_{0}(x)$ and allows us to study finite columns.

When $\tau=0, w(x)=0$ is a solution of (1.1) for all values of $\lambda$. The buckling load for such perfect columns is the lowest bifurcation point of (1.1). This is the lowest value of $\lambda_{0}$ for which solutions of (1.1) with arbitrarily small amplitude exist in every neighborhood of $\lambda_{0}$. A necessary condition for bifurcation to occur is that (1.1) linearized about $\lambda=\lambda_{0}, w(x)=0$ has nontrivial solutions. Thus, bifurcation can occur for $\lambda=\lambda_{0}$ only if

$$
\frac{d^{4} \phi}{d x^{4}}+2 \lambda_{0} \frac{d^{2} \phi}{d x^{2}}+\phi=0, \quad \phi(0)=\phi(\pi)=0, \quad \phi^{\prime \prime}(0)=\phi^{\prime \prime}(\pi)=0
$$

has nontrivial solutions. Clearly, (1.2) has nontrivial solutions only for

$$
\lambda_{0}=\lambda_{k}=\frac{1}{2}\left(k^{2}+\frac{1}{k^{2}}\right), \quad k=1,2, \cdots,
$$

in which case

$$
\phi_{k}(x)=a \sin k x .
$$

Thus the lowest possible bifurcation point is $\lambda=\lambda_{1}=1$.

For $\tau \neq 0$, there is no "trivial" solution of (1.1) for all values of $\lambda$, so that, strictly speaking, bifurcation cannot occur. Instead we define the buckling point to be the lowest point $\lambda_{0}$ for which, in every neighborhood of $\lambda_{0}$, two solutions of (1.1) with arbitrarily small difference exist for the same value of $\lambda$. If $w(x)$ is a solution of (1.1) for $\lambda=\lambda_{0}$, a necessary condition that $\lambda=\lambda_{0}$ is a buckling load is that (1.1) linearized about $w(x)$ have nontrivial solutions. Therefore, the buckling load is defined to be the smallest value of $\lambda$ for which

$\frac{d^{4} \phi}{d x^{4}}+\frac{2 d^{2} \phi}{d x^{2}}+\phi=3 w^{2} \phi+2(1-\lambda) \frac{d^{2} \phi}{d x^{2}}, \quad \phi(0)=\phi(\pi)=0, \phi^{\prime \prime}(0)=\phi^{\prime \prime}(\pi)=0$

has nontrivial solutions, where $w(x)$ and $\lambda$ simultaneously satisfy (1.1).

We solve (1.1) and (1.5) simultaneously for $(w(x), \phi(x), \lambda, \tau)$ in a neighborhood of $\left(0, \phi_{1}, 1,0\right)$ to give us the buckling load as a function of $\tau$. We write (1.1) in the form

$$
\begin{aligned}
\frac{d^{4} w}{d x^{4}}+2 \frac{d^{2} w}{d x^{2}}+w=w^{3}+2(1-\lambda) & \frac{d^{2} w}{d x^{2}}-2 \lambda \tau \frac{d^{2} w_{0}}{d x^{2}}, \\
& w(0)=w(\pi)=0, \quad w^{\prime \prime}(0)=w^{\prime \prime}(\pi)=0,
\end{aligned}
$$

and note that (1.5) and (1.6) are both of the form

$$
d^{4} y / d x^{4}+2\left(d^{2} y / d x^{2}\right)+y=h(x), \quad y(0)=y(\pi)=0, \quad y^{\prime \prime}(0)=y^{\prime \prime}(\pi)=0 .
$$

Since (1.2) has the nontrivial solution $\phi_{1}=a \sin x$, we know, according to the Fredholm alternative theorem, that solutions of (1.7) exist only if $h(x)$ satisfies

$$
\int_{0}^{\pi} h(x) \sin x d x=0 .
$$

If $h(x)$ satisfies (1.8), then all solutions of (1.7) can be expressed as 


$$
y(x)=A \sin x+\int_{0}^{\pi} \mathcal{S}(x, \zeta) h(\zeta) d \zeta,
$$

where $2 / \pi \int_{0}{ }^{x} y(x) \sin x d x=A$. Here $\mathcal{G}(x, \zeta)$ is the generalized Green's function [5, pp. 354-358], which in the present case is

$$
\begin{aligned}
\mathrm{G}(x, \zeta)= & \frac{1}{4 \pi}\left(x^{2}+(\zeta-\pi)^{2}-\frac{1}{3} \pi^{2}-\frac{3}{2}\right) \sin \zeta \sin x+\frac{x}{2 \pi} \sin \zeta \cos x \\
& -\left(\frac{\zeta-\pi}{2 \pi}\right) x \cos \zeta \cos x+\left(\frac{\zeta-\pi}{2 \pi}\right) \cos \zeta \sin x, \quad 0 \leq x<\zeta \leq \pi, \\
= & \left.\frac{1}{4 \pi}(x-\pi)^{2}+\zeta^{2}-\frac{1}{3} \pi^{2}-\frac{3}{2}\right) \sin \zeta \sin x+\frac{\zeta}{2 \pi} \cos \zeta \sin x \\
& -\frac{\zeta}{2 \pi}(x-\pi) \cos \zeta \cos x+\left(\frac{x-\pi}{2 \pi}\right) \sin \zeta \cos x, \quad 0 \leq \zeta<x \leq \pi .
\end{aligned}
$$

Oụr method consists of solving (1.5) and (1.6) by iterations in a neighborhood of the known "trivial" solution $(w(x), \phi(x), \lambda, \tau)=(0, \sin x, 1,0)$. To do this we let the initial iterates be

$$
w^{0}(x, \epsilon)=\epsilon \sin x, \phi^{0}(\epsilon, x)=\sin x,
$$

and define the $\nu+1$ st iterates by

$$
\begin{aligned}
\lambda^{\nu+1}(\epsilon)= & 1-\frac{3}{\pi} \int_{0}^{\pi}\left(w^{\nu}(x, \epsilon)\right)^{2} \phi^{\nu}(x, \epsilon) \sin x d x \\
\tau^{\nu+1}(\epsilon)= & \frac{-1}{\lambda^{\nu+1}(\epsilon) W_{1}}\left[\frac{1}{\pi} \int_{0}^{\pi}\left(w^{\nu}(x, \epsilon)\right)^{3} \sin x d x+\epsilon\left(\lambda^{\nu+1}(\epsilon)-1\right)\right] \\
\phi^{\nu+1}(x, \epsilon)= & \sin x+\int_{0}^{\pi} \oint(x, \zeta)\left[3\left(w^{\nu}(\zeta, \epsilon)\right)^{2} \phi^{\nu}(\zeta, \epsilon)+2\left(1-\lambda^{\nu+1}(\epsilon)\right) \frac{\partial^{2} \phi^{\nu}(\zeta, \epsilon)}{\partial x^{2}}\right] d \zeta \\
w^{\nu+1}(x, \epsilon)= & \epsilon \sin x+\int_{0}^{\pi} \mathcal{S}(x, \zeta)\left[\left(w^{\nu}(\zeta, \epsilon)\right)^{3}+2\left(1-\lambda^{\nu+1}(\epsilon)\right) \frac{\partial^{2} w^{\nu}(\zeta, \epsilon)}{\partial x^{2}}\right. \\
& \left.-2 \lambda^{\nu+1}(\epsilon) \tau^{\nu+1}(\epsilon) \frac{d^{2} w_{0}(\zeta)}{d x^{2}}\right] d \zeta .
\end{aligned}
$$

Here we have assumed that $\int_{0}{ }^{*}\left(d^{2} w_{0}(x) / d x^{2}\right) \sin x d x=-\left(W_{1} \pi / 2\right) \neq 0$. Clearly, $(1.12 \mathrm{a}, \mathrm{b})$ specify $\lambda^{\nu+1}(\epsilon)$ and $\tau^{\nu+1}(\epsilon)$ so that the orthogonality condition (1.8) is satisfied at each iteration. The form of the Green's function $\mathcal{G}(x, \zeta)$ insures us that $\phi^{\nu+1}(x, \epsilon)$ and $w^{\nu+1}(x, \epsilon)$ are four times continuously differentiable whenever $\phi^{\nu}(x, \epsilon)$ and $w^{\nu}(x, \epsilon)$ are also four times continuously differentiable. Therefore, the iteration scheme (1.12) is well defined.

It is easily shown [8] using the contraction mapping principle that this iteration procedure is convergent for all $\epsilon$ in $0<|\epsilon| \leq \epsilon_{0}$ provided $\epsilon_{0}$ is sufficiently small. Furthermore, the limit is the unique solution of (1.5) and (1.6) of the form

$$
\begin{gathered}
w(x, \epsilon)=\epsilon \sin x+\epsilon^{3} w_{1}(x, \epsilon), \quad \phi(x, \epsilon)=\sin x+\epsilon^{2} \phi_{1}(x, \epsilon), \\
\lambda(\epsilon)=1+\epsilon^{2} \lambda_{1}(\epsilon), \quad \tau(\epsilon)=\epsilon^{3} \tau_{1}(\epsilon),
\end{gathered}
$$


where

$$
\int_{0}^{\pi} w_{1}(x, \epsilon) \sin x d x=0, \quad \int_{0} \phi_{1}(x, \epsilon) \sin x d x=0,
$$

and where $w_{1}(x, \epsilon), \phi_{1}(x, \epsilon), \lambda_{1}(\epsilon), \tau_{1}(\epsilon)$ are uniformly bounded on $0<|\epsilon| \leq \epsilon_{0}$. The errors in the $\nu$ th iterate are of the form

$$
\begin{array}{cc}
\left\|w^{\nu}(x, \epsilon)-w(x, \epsilon)\right\|_{\infty}=O\left(\epsilon^{2 v+3}\right), & \left\|\phi^{\nu}(x, \epsilon)-\phi(x, \epsilon)\right\|_{\infty}=O\left(\epsilon^{2 v+2}\right), \\
\left|\lambda^{\nu}(\epsilon)-\lambda(\epsilon)\right|=O\left(\epsilon^{2 v+2}\right), & \left|\tau^{\nu}(\epsilon)-\tau(\epsilon)\right|=O\left(\epsilon^{2 v+3}\right) .
\end{array}
$$

Note that (1.15) implies that the $\nu$ th iterate is asymptotic to the exact solution. Thus we can eliminate $\epsilon$ between $\lambda^{\nu}(\epsilon)$ and $\tau^{\nu}(\epsilon)$ to get an asymptotic representation of the buckling load $\lambda=\lambda(\tau)$ (valid for $\tau \rightarrow 0$ ). Furthermore, by using higher-order iterates, we can determine $\lambda=\lambda(\tau)$ as accurately as we wish for some sufficiently small range of $\tau$.

To explicitly evaluate the iterates, we write $w_{0}(x)$ in the form

$$
w_{0}(x)=\sum_{k=1}^{\infty} W_{k} \sin k x,
$$

and require that $W_{1} \neq 0$. (Note that if $W_{1}=0$, then the iteration scheme (1.11)-(1.12) is not well defined.) The first two iterations now become

$$
\begin{aligned}
\lambda^{1}(\epsilon) & =1-\frac{9}{8} \epsilon^{2}, \\
\tau^{1}(\epsilon) & =\frac{3}{4} \frac{\epsilon^{3}}{W_{1}\left(1-9 / 8 \epsilon^{2}\right)}, \\
\phi^{1}(x, \epsilon) & =\sin x-\frac{3}{256} \epsilon^{2} \sin 3 x \\
w^{1}(x, \epsilon) & =\epsilon \sin x-\epsilon^{3}\left[\frac{3}{2 W} \sum_{k=2}^{\infty} \frac{k^{2} W_{k}}{\left(k^{2}-1\right)^{2}} \sin k x-\frac{1}{256} \sin 3 x\right],
\end{aligned}
$$

and

$$
\begin{aligned}
& \lambda^{2}(\epsilon)=1-\frac{9}{8} \epsilon^{2}+\epsilon^{4}\left(\frac{3}{512}\right)\left[27 \frac{W_{3}}{W_{1}}-\frac{5}{4}\right]+O\left(\epsilon^{6}\right) \\
& \tau^{2}(\epsilon)=\frac{1}{W_{1} \lambda^{2}(\epsilon)}\left[\frac{3}{4} \epsilon^{3}+\epsilon^{5}\left(\frac{3}{1024}\right)\left(27 \frac{W_{3}}{W_{1}}-2\right)\right]+O\left(\epsilon^{7}\right) .
\end{aligned}
$$

Eliminating $\epsilon$ in (1.18), we find

$$
\lambda(\tau)=1-\left(\frac{9}{4 \sqrt{ } 2} W_{1} \tau\right)^{2 / 3}+\frac{1}{256}\left(\frac{4}{3}\right)^{1 / 3}\left(81 \frac{W_{3}}{W_{1}}+\frac{527}{2}\right)\left(W_{1} \tau\right)^{4 / 3}+O\left(\tau^{2}\right) .
$$

The term in (1.19) involving $\tau^{2 / 3}$ is the same as that given in [1] for the special case $W_{1}=1$ and $W_{k}=0$ for $k \neq 1$. In addition, (1.19) gives us the $\tau^{4 / 3}$ correction term and shows the effect of the third harmonic $W_{3}$. Additional harmonics appear in higher-order terms which are easily obtained by continuing the iteration procedure.

If we know the distribution of the imperfections for some ensemble of imperfect columns, we can compute ensemble averages of the load-imperfection relation. That is, since (1.19) is valid for each member of the ensemble, we find the expected value of the buckling load to be 


$$
\langle\lambda(\tau)\rangle=1-\tau^{2 / 3}\left(\frac{9}{4 \sqrt{ } 2}\right)^{2 / 3}\left\langle W^{2 / 3}\right\rangle+\frac{\tau^{4 / 3}}{128}\left(\frac{1}{6}\right)^{1 / 3}\left(81\left\langle W_{3} W_{1}^{1 / 3}\right\rangle+\frac{527}{2}\left\langle W_{1}^{4 / 3}\right\rangle\right)+\cdots
$$

This can be compared with other approaches for random imperfections as in [1] or [2].

Through each of the solutions (1.13) of (1.5) and (1.6) with $\epsilon$ fixed in $0<|\epsilon| \leq \epsilon_{0}$ we can determine a unique solution of (1.1) on which $\tau$ is a nonzero constant. We write (1.1) as

$$
\begin{array}{r}
\frac{d^{4} \psi}{d x^{4}}+2 \lambda(\epsilon) \frac{d^{2} \psi}{d x^{2}}+\left(1-3 w^{2}(x, \epsilon)\right) \psi=3 w(x, \epsilon) \psi^{2}+\psi^{3} \\
-2(\lambda-\lambda(\epsilon))\left(\tau(\epsilon) \frac{d^{2} w_{0}}{d x^{2}}+\frac{d^{2} w(x, \epsilon)}{d x^{2}}+\frac{d^{2} \psi}{d x^{2}}\right), \\
\psi(0)=\psi(\pi)=0, \quad \psi^{\prime \prime}(0)=\psi^{\prime \prime}(\pi)=0,
\end{array}
$$

where $\psi=w-w(x, \epsilon)$ and where $w(x, \epsilon), \lambda(\epsilon)$ and $\tau(\epsilon)$ are the solutions of (1.1) given by (1.13). Eq. (1.21) is of the form

$\frac{d^{4} y}{d x^{4}}+2 \lambda(\epsilon) \frac{d^{2} y}{d x^{2}}+\left(1-3 w^{2}(x, \epsilon)\right) y=h(x), \quad y(0)=y(\pi)=0, \quad y^{\prime \prime}(\pi)=y^{\prime \prime}(\pi)=0$.

Since (1.6) has the nontrivial solution $\phi(x, \epsilon)$ given in (1.13), we know that solutions of (1.21) exist only if

$$
\int_{0}^{\pi} h(x) \phi(x, \epsilon) d x=0 .
$$

If $h(x)$ satisfies (1.22), and $\epsilon_{0}$ is chosen sufficiently small that $\phi(x, \epsilon)$ is the only eigenfunction of (1.5), then all solutions of (1.21) can be expressed as

$$
y(x)=A \phi(x, \epsilon)+\int_{0}^{\pi} \mathcal{G}_{\epsilon}(x, \zeta) h(\zeta) d \zeta
$$

where $\int_{0}{ }^{\pi}(y(x)-A \phi(x, \epsilon)) \phi(x, \epsilon) d x=0$. Here $\mathcal{G}_{\epsilon}(x, \zeta)$ is the appropriate generalized Green's function for (1.21) which can be calculated in principal.

We find solutions of (1.21) by iterations in a neighborhood of the known solution (1.13). To do this we let the initial iterate be

$$
w^{0}(x, \epsilon, \delta)=w(x, \epsilon)+\psi^{0}(x, \epsilon, \delta), \quad \psi^{0}(x, \epsilon, \delta)=\delta \phi(x, \epsilon)
$$

and define the $\nu+1$ st iterate by

$$
\begin{aligned}
\lambda^{\nu+1}(\epsilon, \delta) & =\lambda(\epsilon)+\frac{1}{2 \Phi^{\nu}(\epsilon, \delta)} \int_{0}^{\pi}\left[3 w(x, \epsilon)\left(\psi^{\nu}(x, \epsilon, \delta)\right)^{2}+\left(\psi^{\nu}(x, \epsilon, \delta)\right)^{3}\right] \phi(x, \epsilon) d x, \\
\Phi^{\nu}(\epsilon, \delta)= & \int_{0}^{\pi} \phi(x, \epsilon) \frac{d^{2}}{d x^{2}}\left(\tau(\epsilon) w_{0}(x)+w(x, \epsilon)+\psi^{\nu}(x, \epsilon, \delta)\right) d x, \\
\psi^{\nu+1}(x, \epsilon, \delta)= & \delta \phi(x, \epsilon)+\int_{0}^{\pi} \oint_{\epsilon}(x, \zeta)\left[3 w(\zeta, \epsilon)\left(\psi^{\nu}(\zeta, \epsilon, \delta)\right)^{2}+\left(\psi^{\nu}(\zeta, \epsilon, \delta)\right)^{3}\right. \\
& \left.-2\left(\lambda^{\nu+1}-\lambda(\epsilon)\right) \frac{d^{2}}{d x^{2}}\left(\tau(\epsilon) w_{0}(\zeta)+w(\zeta, \epsilon)+\psi^{\nu}(\zeta, \epsilon, \delta)\right)\right] d \zeta
\end{aligned}
$$


It is easily shown that the iteration scheme (1.25) is well defined and is convergent for all $\delta$ in $0<|\delta| \leq \delta_{0}(\epsilon)$ provided $\delta_{0}(\epsilon)$ is sufficiently small. Furthermore, the limit is the unique solution of (1.1) of the form

$$
w(x, \epsilon, \delta)=w(x, \epsilon)+\delta \phi(x, \epsilon)+\delta^{2} w_{2}(x, \epsilon, \delta), \lambda(\epsilon, \delta)=\lambda(\epsilon)+\delta^{2} \mu(\epsilon, \delta),
$$

where $w_{2}(x, \epsilon, \delta)$ and $\mu(\epsilon, \delta)$ are uniformly bounded on $0<|\delta| \leq \delta_{0}(\epsilon)$. The errors in the $\nu$ th iterate are of the form

$$
\left\|w^{\nu}(x, \epsilon, \delta)-w(x, \epsilon, \delta)\right\|_{\infty}=O\left(\delta^{\nu+2}\right),\left|\lambda^{\nu}(\epsilon, \delta)-\lambda(\epsilon, \delta)\right|=O\left(\delta^{\nu+2}\right) .
$$

Since this implies that the $\nu$ th iterate is asymptotic to the exact solution, we find the form of the solution by examining $\lambda^{1}(\epsilon, \delta)$. Applying (1.24) and (1.25), we find

$$
\lambda^{1}(\epsilon, \delta)=\lambda(\epsilon)-\frac{3}{8} \delta^{2}\left[\left(\frac{3+\delta / \epsilon}{1+\delta / \epsilon}\right)+O\left(\epsilon^{2}\right)\right] .
$$

Combining this with (1.18), we see that

$$
\lambda(\epsilon, \delta)=\lambda_{0}-\frac{9}{8}\left(\epsilon^{2}+\delta^{2}\left(\frac{1+\delta / 3 \epsilon}{1+\delta / \epsilon}\right)\right)+\cdots,
$$

which is a representation of the load-displacement curve when $\tau=\tau(\epsilon)$ in a neighborhood of $\lambda_{0}$. Clearly, $\lambda(\epsilon, \delta)$ is maximized when $\delta=0$, so that $\lambda=\lambda(\epsilon, 0)$ is the buckling load in the sense of other authors [2, 7] as well. A plot of (1.29) is shown in Fig. 1.

II. Buckling of a spherical cap. In a given physical problem, the buckling load may be sensitive to a number of different parameters which are either naturally occurring or artifically introduced as, for example, in [11]. In this section we will consider the effect of general axisymmetric imperfections on the buckling load of a spherical cap, and we will also investigate the change in buckling load resulting from a change of the boundary constraints of the cap.

Suppose the surface of a shallow, thin elastic spherical cap is subjected to a uniform

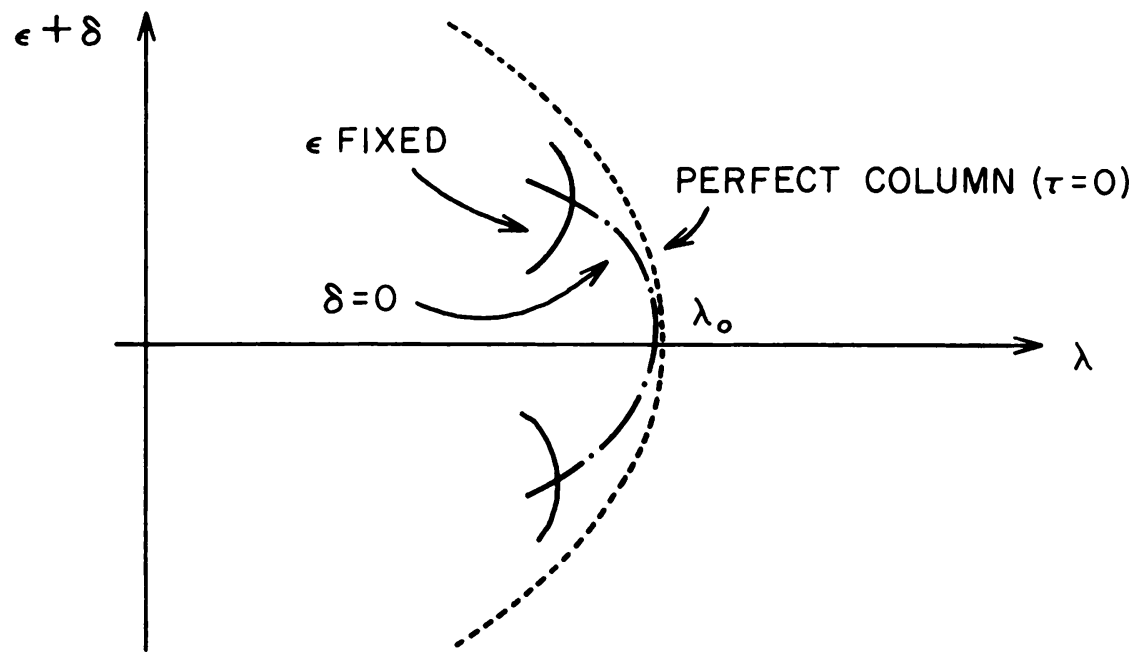

Fig. 1. Plot of load-displacement, curve for column (Eq. (1.29)). 
pressure directed toward the cap's center of curvature. The nondimensional equations for axisymmetric deformations of this initially imperfect cap are [3]

$$
L W=\rho\left(W V+P x^{2}+\tau V W_{0}\right), L V=\rho\left(x^{2}-W^{2}-2 \tau W_{0}(W-x)\right) .
$$

Here $W(x)$ is proportional to the slope of the tangent plane of the deformed surface, $V(x)$ is a stress function, and $L u=x(d / d x)(1 / x(d / d x)(x u))$. The other dimensionless quantities are

$$
\begin{aligned}
& x=r / a, \quad 0 \leq r \leq a, \quad W_{0}(x)=(R / a)\left(d w_{0}(r) / d r\right), \\
& \rho=\frac{2}{C}\left(\frac{a}{t}\right)\left(\frac{a}{R}\right), \quad P=\frac{2}{C E}\left(\frac{R}{t}\right)^{2} p, \quad C^{2}=\frac{2}{3\left(1-\nu^{2}\right)},
\end{aligned}
$$

where $a, R$ and $t$ are the radius of the base, the radius of curvature and shell thickness, respectively, and $w_{0}(r)$ is the initial slope of the imperfection with amplitude $\tau$. The uniform pressure is $p$. Requiring regularity and symmetry at $x=0$ and allowing no rotation at $x=1$ gives the edge constraints

$$
W(0)=V(0)=0, W(1)=1 .
$$

An additional edge constraint must be imposed at $x=1$. We will consider two possibilities:

$$
\begin{gathered}
V^{\prime}(1)-\nu V(1)=\left(\xi \alpha_{1}-1\right)(1-\nu) P, \quad \tau=\xi \alpha_{2}, \quad \alpha_{1}^{2}+\alpha_{2}^{2}=1, \\
V(1)=-P,
\end{gathered}
$$

Physically, these conditions imply that the meridional displacement in (2.4a) or the meridional stress in (2.4b) is proportional to the external pressure. When $\xi \alpha_{1}=1,(2.4 \mathrm{a})$ implies that the edge of the shell is clamped, while when $\xi \alpha_{1}=0$, the edge is allowed to move freely in radial directions. For $\xi \alpha_{1}$ between 0 and 1 there are certain elastic restoring forces applied at the edge. Finally, by appropriate choices of $\alpha_{1}$ and $\alpha_{2}$, we can examine the effects of combinations of imperfections and changing boundary constraints on the buckling load. The bifurcation problem $(\tau=\xi=0)$ for $(2.1),(2.3)$ and $(2.4 \mathrm{a}, \mathrm{b})$ has been treated in [13]. It is also possible to treat the bifurcation problem using an iteration procedure similar to the method used here, as, for instance, in [10] and [12].

We first consider (2.1) subject to the edge constraints (2.3) and (2.4a). A uniform compression, $W(x)=x, V(x)=\left(\xi \alpha_{1}-1\right) P x$, satisfies the edge constraints, and satisfies the equation for $\xi=0$. With the transformation

$$
W(x)=x+w(x), \quad V(x)=\left(\xi \alpha_{1}-1\right) P x+v(x),
$$

the problem becomes

$$
\begin{gathered}
\left(\begin{array}{ll}
L & 0 \\
0 & L
\end{array}\right)\left(\begin{array}{l}
w \\
v
\end{array}\right)+\rho x\left(\begin{array}{cc}
P_{0} & -1 \\
2 & 0
\end{array}\right)\left(\begin{array}{l}
w \\
v
\end{array}\right)=\rho\left(\begin{array}{c}
\left(P_{0}-P\right) x w+w v+\xi g(P, w, v)+\xi^{2} h(P) \\
-w^{2}-\xi f(P, w, v)
\end{array}\right) \\
=\left(\begin{array}{c}
R_{1}(P, \xi, w, v)+\xi^{2} h(P) \\
R_{2}(P, \xi, w, v)
\end{array}\right) \\
w(0)=v(0)=w(1)=v^{\prime}(1)-v v(1)=0
\end{gathered}
$$


where

$$
\begin{aligned}
& g(P, w, v)=\alpha_{1} P x(w(x)+x)+\alpha_{2} W_{0}(x)(v(x)-P x), \\
& h(P)=\alpha_{1} \alpha_{2} P x W_{0}(x), \quad f(P, w, v)=2 \alpha_{2} W_{0}(x) w(x),
\end{aligned}
$$

and $P_{0}$ is arbitrary.

As in Sec. I, if $w(x)$ and $v(x)$ are a solution of (2.6) at the "buckling" load, then (2.6) linearized about this solution must possess nontrivial solutions. Therefore, we seek nontrivial solutions of

$$
\begin{aligned}
\left(\begin{array}{ll}
L & 0 \\
0 & L
\end{array}\right)\left(\begin{array}{l}
\varphi \\
\psi
\end{array}\right)+\rho x\left(\begin{array}{rr}
P_{0} & -1 \\
2 & 0
\end{array}\right)\left(\begin{array}{l}
\varphi \\
\psi
\end{array}\right) \\
\quad=\rho\left(\begin{array}{cc}
\left(P_{0}-P\right) x+v+\xi g_{v}(P, w, v) & w+\xi g_{v}(P, w, v) \\
-2 w-\xi f_{w}(P, w, v) & -\xi f_{v}(P, w, v)
\end{array}\right)\left(\begin{array}{l}
\varphi \\
\psi
\end{array}\right) \\
=\left(\begin{array}{c}
R_{3}(P, \xi, w, v, \varphi, \psi) \\
R_{4}(P, \xi, w, v, \varphi, \psi)
\end{array}\right), \quad \varphi(0)=\psi(0)=\varphi(1)=\psi^{\prime}(1)-\nu \psi(1)=0 .
\end{aligned}
$$

When $\xi=w(x)=v(x)=0,(2.7)$ is an eigenvalue problem which determines the possible bifurcation values of $P$. The eigenvalues and eigenvectors are

$$
\begin{aligned}
& P_{0_{n}}=\left(\lambda_{n}^{2} / \rho\right)+\left(2 \rho / \lambda_{n}^{2}\right), \\
& {\left[\begin{array}{l}
\varphi_{0} \\
\psi_{0}
\end{array}\right]_{n}=}\left(\begin{array}{c}
J_{1}\left(\frac{\sqrt{ } 2 \rho}{\lambda_{n}}\right) J_{1}\left(\lambda_{n}\right)-J_{1}\left(\lambda_{n}\right) J_{1}\left(\frac{\sqrt{ } 2 \rho}{\lambda_{n}} x\right) \\
-\frac{\rho}{\lambda_{n}{ }^{2}} J_{1}\left(\frac{\sqrt{ } 2 \rho}{\lambda_{n}}\right) J_{1}\left(\lambda_{n} x\right)-J_{1}\left(\lambda_{n}\right) J_{1}\left(\frac{\sqrt{ } 2 \rho}{\lambda_{n}} x\right)
\end{array}\right), \\
&\left(\begin{array}{l}
\varphi_{0}^{*} \\
\psi_{0}^{*}
\end{array}\right]_{n}=\left(\begin{array}{c}
J_{1}\left(\frac{\sqrt{ } 2 \rho}{\lambda_{n}}\right) J_{1}\left(\lambda_{n} x\right)-J_{1}\left(\lambda_{n}\right) J_{1}\left(\frac{\sqrt{ } 2 \rho}{\lambda_{n}} x\right) \\
-\frac{\rho}{\lambda_{n}{ }^{2}} J_{1}\left(\frac{\sqrt{ } 2 \rho}{\lambda_{n}}\right) J_{1}\left(\lambda_{n} x\right)-\frac{\lambda_{n}{ }^{2}}{2 \rho} J_{1}\left(\lambda_{n}\right) J_{1}\left(\frac{\sqrt{ } 2 \rho}{\lambda_{n}} x\right)
\end{array}\right),
\end{aligned}
$$

where the numbers $\lambda_{n}$ are roots of the transcendental equation

$0=\frac{\sqrt{ } 2 \rho}{\lambda^{2}} J_{1}\left(\frac{\sqrt{ } 2 \rho}{\lambda_{n}}\right)\left(\lambda J_{1}{ }^{\prime}(\lambda)-\nu J_{1}(\lambda)\right)-\frac{\lambda^{2}}{\sqrt{ } 2 \rho} J_{1}(\lambda)\left(\frac{\sqrt{ } 2 \rho}{\lambda} J_{1}{ }^{\prime}\left(\frac{\sqrt{ } 2 \rho}{\lambda}\right)-\nu J_{1}\left(\frac{\sqrt{ } 2 \rho}{\lambda}\right)\right)$,

and where $\left(\begin{array}{c}\varphi_{0} 0^{*} \\ \psi_{0}^{*}\end{array}\right)$ is the solution of corresponding adjoint equation. Since we are interested in the smallest value of $P$ for which nontrivial solutions of (2.7) exist, the bifurcation point $P_{0}$ of interest is that value of $P_{0_{n}}$ which minimizes $P_{0_{n}}=\left({\lambda_{n}}^{2} / \rho\right)+\left(2 \rho / \lambda_{n}{ }^{2}\right)$.

As in Sec. I, the Fredholm alternative theorem is applicable for finding solutions of (2.6) and (2.7). Specifically, since the problem (2.7) is not selfadjoint when $\xi=w(x)=$ $v(x)=0$, Eqs. (2.6) and (2.7) have solutions only if their right-hand sides, say $\left(\begin{array}{c}Q(x) \\ R(x)\end{array}\right)$, satisfy

$$
\int_{0}^{1}\left(\varphi_{0}^{*}(x) Q(x)+\psi_{0}^{*}(x) R(x)\right) d x=0
$$


where $\varphi_{0}{ }^{*}(x)$ and $\psi_{0}{ }^{*}(x)$ are the components of the adjoint eigenfunction corresponding to $P_{0}$. If (2.9) is satisfied, then solutions of (2.6) and (2.7) are given in terms of the generalized Green's matrix $G(x, \zeta)$ as

$$
\left(\begin{array}{l}
w(x) \\
v(x)
\end{array}\right)=A\left(\begin{array}{l}
\varphi_{0}(x) \\
\psi_{0}(x)
\end{array}\right)+\int_{0}^{1} G(x, \zeta)\left(\begin{array}{l}
Q(\zeta) \\
R(\zeta)
\end{array}\right) d \zeta
$$

The Green's matrix for this problem is given in the Appendix.

As in Sec. I, we propose an iteration scheme with initial iterate

$$
\begin{aligned}
w^{0}(x, \epsilon) & =\epsilon \varphi_{0}(x), & & v^{0}(x, \epsilon)=\epsilon \psi_{0}(x), \\
\varphi^{0}(x, \epsilon) & =\varphi_{0}(x), & & \psi^{0}(x, \epsilon)=\psi_{0}(x), \\
P^{0}(\epsilon) & =P_{0}, & & \xi^{0}(\epsilon)=0 .
\end{aligned}
$$

The $\nu+1$ st iterate is defined by

$$
\begin{aligned}
& \int_{0}^{1}\left[\varphi_{0}^{*}(x) R_{1}\left(P^{\nu+1}(\epsilon), \xi^{\nu+1}(\epsilon), w^{\nu}(x, \epsilon), v^{\nu}(x, \epsilon)\right)+\varphi_{0}^{*}(x)\left(\xi^{\nu}(\epsilon)\right)^{2} h\left(P^{\nu}(\epsilon)\right)\right. \\
& \left.+\psi_{0}^{*}(x) R_{2}\left(P^{\nu+1}(\epsilon), \xi^{\nu+1}(\epsilon), w^{\nu}(x, \epsilon), v^{\nu}(x, \epsilon)\right)\right] d x=0, \\
& \int_{0}^{1}\left[\varphi_{0}^{*}(x) R_{3}\left(P^{\nu+1}(\epsilon), \xi^{\nu+1}(\epsilon), w^{\nu}(x, \epsilon), v^{\nu}(x, \epsilon), \varphi^{\nu}(x, \epsilon), \psi^{\nu}(x, \epsilon)\right)\right. \\
& \left.+\psi_{0}^{*}(x) R_{4}\left(P^{\nu+1}(\epsilon), \xi^{\nu+1}(\epsilon), w^{\nu}(x, \epsilon), v^{\nu}(x, \epsilon), \varphi^{\nu}(x, \epsilon), \psi^{\nu}(x, \epsilon)\right)\right] d x=0 \\
& \left(\begin{array}{l}
w^{\nu+1}(x, \epsilon) \\
v^{\nu+1}(x, \epsilon)
\end{array}\right)=\epsilon\left(\begin{array}{l}
\varphi_{0}(x) \\
\psi_{0}(x)
\end{array}\right) \\
& +\int_{0}^{1} G(x, \zeta)\left[\begin{array}{l}
R_{1}\left(P^{\nu+1}(\epsilon), \xi^{\nu+1}(\epsilon), w^{\nu}(\zeta, \epsilon), v^{\nu}(\zeta, \epsilon)\right)+\left(\xi^{\nu}(\epsilon)\right)^{2} h\left(P^{\nu}(\epsilon)\right. \\
R_{2}\left(P^{\nu+1}(\epsilon), \xi^{\nu+1}(\epsilon), W^{\nu}(\zeta, \nu), v^{\nu}(\zeta, \epsilon)\right)
\end{array}\right] d \zeta, \\
& \left(\begin{array}{l}
\varphi^{\nu}(x, \epsilon) \\
\psi^{\nu}(x, \epsilon)
\end{array}\right)=\left(\begin{array}{l}
\varphi_{0}(x) \\
\psi^{\nu}(x)
\end{array}\right) \\
& +\int_{0}^{1} G(x, \zeta)\left(\begin{array}{l}
R_{3}\left(P^{\nu+1}(\epsilon), \xi^{\nu+1}(\epsilon), w^{\nu}(\zeta, \epsilon), v^{\nu}(\zeta, \epsilon), \varphi^{\nu}(\zeta, \epsilon), \psi^{\nu}(\zeta, \epsilon)\right) \\
R_{4}\left(P^{\nu+1}(\epsilon), \xi^{\nu+1}(\epsilon), w^{\nu}(\zeta, \epsilon), v^{\nu}(\zeta, \epsilon), \varphi^{\nu}(\zeta, \epsilon), \psi^{\nu}(\zeta, \epsilon)\right)
\end{array}\right) d \zeta
\end{aligned}
$$

The iteration scheme (2.12) is well-defined, and it can be shown that for all $\epsilon$ in $0<|\epsilon| \leq \epsilon_{0}$, with $\epsilon_{0}$ sufficiently small, the iteration procedure is convergent to a unique solution of (2.6) and (2.7) of the form

$$
\begin{aligned}
{\left[\begin{array}{l}
w(x, \epsilon) \\
v(x, \epsilon)
\end{array}\right] } & =\epsilon\left(\begin{array}{l}
\varphi_{0}(x) \\
\psi_{0}(x)
\end{array}\right)+\epsilon^{2}\left(\begin{array}{l}
w_{1}(x, \epsilon) \\
v_{1}(x, \epsilon)
\end{array}\right), \\
{\left[\begin{array}{l}
\varphi(x, \epsilon) \\
\psi(x, \epsilon)
\end{array}\right) } & =\left[\begin{array}{l}
\varphi_{0}(x) \\
\psi_{0}(x)
\end{array}\right)+\epsilon\left(\begin{array}{l}
\varphi_{1}(x, \epsilon) \\
\psi_{1}(x, \epsilon)
\end{array}\right), \\
P(\epsilon) & =P_{0}+\epsilon P_{1}(\epsilon), \\
\xi(\epsilon) & =\epsilon^{2} \xi_{1}(\epsilon) .
\end{aligned}
$$


Here

$$
\begin{aligned}
& \int_{0}^{1} x\left(\varphi_{0}^{*}(x) w_{1}(x, \epsilon)+\psi_{0}^{*}(x) v_{1}(x, \epsilon)\right) d x=0 \\
& \int_{0}^{1} x\left(\varphi_{0}^{*}(x) \varphi_{1}(x, \epsilon)+\psi_{0}^{*}(x) \psi_{1}(x, \epsilon)\right) d x=0
\end{aligned}
$$

and $w_{1}(x, \epsilon), v_{1}(x, \epsilon), \varphi_{1}(x, \epsilon), \psi_{1}(x, \epsilon), P_{1}(\epsilon), \xi_{1}(\epsilon)$ are uniformly bounded on $0<|\epsilon| \leq \epsilon_{0}$. The error in the $\nu$ th iterate satisfies

$$
\begin{array}{rlrl}
\left\|w^{\nu}(x, \epsilon)-w(x, \epsilon)\right\|_{\infty} & =O\left(\epsilon^{\nu+2}\right), & \left\|v^{\nu}(x, \epsilon)-v(x, \epsilon)\right\|_{\infty} & =O\left(\epsilon^{\nu+2}\right), \\
\left\|\varphi^{\nu}(x, \epsilon)-\varphi(x, \epsilon)\right\|_{\infty} & =O\left(\epsilon^{\nu+1}\right), & \left\|\psi^{\nu}(x, \epsilon)-\psi(x, \epsilon)\right\|_{\infty} & =O\left(\epsilon^{\nu+1}\right), \\
\left|P^{\nu}(\epsilon)-P(\epsilon)\right| & =O\left(\epsilon^{\nu+1}\right), & \left|\xi^{\nu}(\epsilon)-\xi(\epsilon)\right|=O\left(\epsilon^{\nu+2}\right) .
\end{array}
$$

The first iterate yields

$$
\begin{aligned}
& P^{1}(\epsilon)=P_{0}+2 \epsilon P_{1}+\epsilon^{2} \frac{P_{1}}{P_{0}} \frac{W_{2}}{W_{1}}+\alpha_{2} O\left(\epsilon^{3}\right), \\
& \xi^{1}(\epsilon)=\frac{\epsilon^{2} Q}{P^{1}} \frac{1 \epsilon W_{1}}{}
\end{aligned}
$$

where

$$
\begin{aligned}
Q & =\int_{0}^{1} \varphi_{0}{ }^{2}(x)\left(\psi_{0}(x)-\psi_{0}{ }^{*}(x)\right) d x, \\
P_{1} & =Q / \int_{0}^{1} x \varphi_{0}{ }^{2}(x) d x \\
W_{1} & =\alpha_{1} \int_{0}^{1} x^{2} \varphi_{0}^{*}(x) d x-\alpha_{2} \int_{0}^{1} x W_{0}(x) \varphi_{0}^{*}(x) d x, \\
W_{2} & =\int_{0}^{1} W_{0}(x) \varphi_{0}(x)\left(\psi_{0}(x)-2 \psi_{0}^{*}(x)\right) d x .
\end{aligned}
$$

In Table I we present some values of $P_{0}, Q$ and $P_{1}$ for various values of the physical parameter which were determined with Young's modulus chosen as $\nu=0.3$ by numerical evaluation of the appropriate integrals.

TABLE I

Values of the coefficients of (2.15) for the boundary-value problem (2.1), (2.3), (2.4a). $P_{2}=\int_{0}{ }^{1} x^{2} \varphi_{0}{ }^{*}(x) d x$.

\begin{tabular}{cccccl}
\hline$\sqrt{ } \rho$ & $\lambda_{n}$ & $P_{0}$ & $P_{1}$ & $Q$ & \multicolumn{1}{c}{$P_{2}$} \\
\hline 1.0 & 3.882 & 15.21 & 0.119 & 0.000318 & 0.0190 \\
2.0 & 4.591 & 5.65 & 1.47 & 0.0392 & 0.0551 \\
3.0 & 6.111 & 4.63 & 2.05 & 0.0413 & 0.0206 \\
4.0 & 6.780 & 3.49 & 0.696 & 0.00159 & 0.000697 \\
5.0 & 7.628 & 3.19 & -1.36 & -0.00686 & 0.000901 \\
6.0 & 9.037 & 3.15 & -2.16 & -0.0143 & 0.00107 \\
7.0 & 10.078 & 3.04 & -0.222 & -0.000018 & 0.00000804 \\
8.0 & 11.164 & 2.97 & 1.59 & 0.00542 & 0.000249 \\
9.0 & 12.497 & 2.96 & 1.48 & 0.00309 & 0.000142 \\
10.0 & 13.538 & 2.94 & -0.390 & -0.0000667 & 0.00000795 \\
\hline
\end{tabular}


Eliminating $\epsilon$ in (2.15) gives

$$
P_{ \pm}(\xi)=P_{0} \pm 2 P_{1}\left(\frac{-\xi P_{0} W_{1}}{Q}\right)^{1 / 2}+O(\xi) .
$$

Here $P_{-}(\xi)$ is the imperfection-sensitive buckling load and $P_{+}(\xi)$ corresponds to an "unbuckling" load. Of course, we must require that $\xi P_{0} W_{1} / Q<0$. For each value of the physical parameter $\rho$, this places a restriction on the sign of the "imperfection" $W_{1}$ for which (2.16) is valid. Problems for which $\xi$ is of the opposite sign give rise to buckling-load solutions which do not have nontrivial solutions of (2.7), and therefore are not locally maximal solutions on the load-deflection curve. Instead, the buckling load must be defined in some other way.

We recall that this did not occur in Sec. I. For the buckling load of a column, $\tau(\epsilon)$ in (1.18) is an odd function of $\epsilon$, and $\lambda(\epsilon)$ is an even function of $\epsilon$. Thus, changing the sign of the imperfection does not affect the buckling load. Physically this is clear because of the symmetry of the column. However, one does not expect this to be the case for the spherical cap, since symmetry is lost due to the initial curvature of the perfect cap.

Through each of the solutions (2.13) of (2.6) and (2.7) with $\epsilon$ fixed in $0<|\epsilon| \leq \epsilon_{0}$ we can again determine a unique solution branch of (2.1) on which $\xi$ is a nonzero constant. Following the procedure given in section I we write (2.1) as

$$
\begin{aligned}
& \left(\begin{array}{ll}
L & 0 \\
0 & L
\end{array}\right)\left(\begin{array}{l}
\Phi \\
\Theta
\end{array}\right)+\rho\left(\begin{array}{lc}
P(\epsilon) x-v(x, \epsilon)-\xi(\epsilon) \alpha_{1} P(\epsilon) x & -x-w(x, \epsilon)-\xi(\epsilon) \alpha_{2} W_{0} \\
2 x+2 w(x, \epsilon)+2 \xi(\epsilon) \alpha_{2} W_{0}(x) & 0
\end{array}\right)\left(\begin{array}{l}
\Phi \\
\Theta
\end{array}\right) \\
& =\rho\left(\begin{array}{c}
\Phi \Theta-q\left[x w(x, \epsilon)+x \Phi-\xi(\epsilon)\left(\alpha_{1} x(w(x, \epsilon)+\Phi+x)-\alpha_{2} x W_{0}(x)\right)-\xi^{2}(\epsilon) \alpha_{1} \alpha_{2} x W_{0}(x)\right] \\
-\Phi^{2}
\end{array}\right) \text {, } \\
& \Phi(0)=\Phi(1)=\Theta(0)=\Theta^{\prime}(1)-\nu \Theta(1)=0,
\end{aligned}
$$

where $w=w(x, \boldsymbol{\epsilon})+\Phi, v=v(x, \boldsymbol{\epsilon})+\Theta, P=P(\boldsymbol{\epsilon})+q$ and $w(x, \boldsymbol{\epsilon}), v(x, \boldsymbol{\epsilon}), P(\boldsymbol{\epsilon}), \xi(\boldsymbol{\epsilon})$ are solutions of (2.6), (2.7) as given by (2.13).

Using (2.6b) and (2.7), it is easy to see that the left-hand side of (2.17) has the nontrivial solution $\left(\begin{array}{l}\varphi(x, \epsilon) \\ \psi(x, \epsilon)\end{array}\right)$ as given in (2.13). Therefore, we can use the Fredholm alternative theorem to solve (2.17) by iterations. Let the initial iterate be

$$
\begin{aligned}
w^{0}(x, \epsilon, \delta) & =w(x, \epsilon)+\Phi^{0}(x, \epsilon, \delta), & \Phi^{0}(x, \epsilon, \delta) & =\delta \varphi(x, \epsilon), \\
v^{0}(x, \epsilon, \delta) & =v(x, \epsilon)+\Theta^{0}(x, \epsilon, \delta), & \Theta^{0}(x, \epsilon, \delta) & =\delta \psi(x, \epsilon),
\end{aligned}
$$

and define the $\nu+1$ st iterate by

$$
\begin{aligned}
Q^{\nu}(x, \epsilon, \delta)= & x w(x, \epsilon)+x \Phi^{\nu}(x, \epsilon, \delta) \\
& -\xi(\epsilon)\left(\alpha_{1} x\left(w(x, \epsilon)+\Phi^{\nu}(x, \epsilon, \delta)+x\right)-\alpha_{2} x W_{0}(x)\right)-\xi^{2}(\epsilon) \alpha_{1} \alpha_{2} x W_{0}(x), \\
q^{\nu+1}(\epsilon, \delta)= & \frac{\int_{0}^{1}\left[\varphi^{*}(x, \epsilon) \Phi^{\nu}(x, \epsilon, \delta) \Theta^{\nu}(x, \epsilon, \delta)-\psi^{*}(x, \epsilon)\left(\Theta^{\nu}(x, \epsilon, \delta)\right)^{2}\right] d x}{\int_{0}^{1} \varphi^{*}(x, \epsilon) Q^{\nu}(x, \epsilon, \delta) d x}, \quad(2.18 \mathrm{~b}) \\
\left(\begin{array}{c}
\Phi^{\nu+1}(x, \epsilon, \delta) \\
\Theta^{\nu+1}(x, \epsilon, \delta)
\end{array}\right]= & \rho \int_{0}^{1} G_{\epsilon}(x, \zeta)\left[\begin{array}{c}
\Phi^{\nu}(\zeta, \epsilon, \delta) \Theta^{\nu}(\zeta, \epsilon, \delta)-q^{\nu+1}(\epsilon, \delta) Q^{\nu}(\zeta, \epsilon, \delta) \\
-\left(\Phi^{\nu}(\zeta, \epsilon, \delta)\right)^{2}
\end{array}\right] d \zeta
\end{aligned}
$$


where $G_{\epsilon}(x, \zeta)$ is the appropriate generalized Green's matrix for $(2.17)$, and $\varphi^{*}(x, \epsilon)$, $\psi^{*}(x, \epsilon)$ are components of the solution of the adjoint of (2.7).

It is easily shown that the iteration scheme (2.18) is well defined and is convergent for all $\delta$ in $0<|\delta| \leq \delta_{0}(\epsilon)$ provided $\delta_{0}(\epsilon)$ is sufficiently small. The limit is the unique solution of $(2.1)$ of the form

$$
\begin{aligned}
w(x, \epsilon, \delta) & =w(x, \epsilon)+\delta \varphi(x, \epsilon)+\delta^{2} w_{2}(x, \epsilon, \delta), \\
v(x, \epsilon, \delta) & =v(x, \epsilon)+\delta \psi(x, \epsilon)+\delta^{2} v_{2}(x, \epsilon, \delta), \\
P(\epsilon, \delta) & =P(\epsilon)+\delta^{2} q(\epsilon, \delta),
\end{aligned}
$$

where $w_{2}(x, \epsilon, \delta), v_{2}(x, \epsilon, \delta)$ and $\mu(\epsilon, \delta)$ are uniformly bounded on $0<|\delta| \leq \delta_{0}(\epsilon)$, and $\int x\left(\varphi^{*}(x, \epsilon) w_{2}(x, \epsilon, \delta)+\psi^{*}(x, \epsilon) v_{2}(x, \epsilon, \delta)\right) d x=0$. The errors in the $v$ th iterate are of the form

$$
\begin{gathered}
\left\|w^{\nu}(x, \epsilon, \delta)-w(x, \epsilon, \delta)\right\|_{\infty}=O\left(\delta^{\nu+2}\right), \quad\left\|v^{\nu}(x, \epsilon, \delta)-v(x, \epsilon, \delta)\right\|_{\infty}=O\left(\delta^{\nu+2}\right), \\
\left|P^{\nu}(\epsilon, \delta)-P(\epsilon, \delta)\right|=O\left(\delta^{\nu+2}\right) .
\end{gathered}
$$

We find the asymptotic form of $P(\epsilon, \delta)$ by examining $P^{1}(\epsilon, \delta)$. Applying (2.18), we find

$$
P^{1}(\epsilon, \delta)=P(\epsilon)+\delta^{2}\left(\frac{P_{1}}{\epsilon+\delta}+O(1)\right) \text { for }|\delta|<|\epsilon| .
$$

Combining this with (2.15), we see that

$$
P(\epsilon, \delta)=P_{0}+\epsilon P_{1}\left(1+\frac{\delta^{2}}{\epsilon^{2}} \frac{1}{1+\delta / \epsilon}\right)+\cdots, \quad \xi(\epsilon)=\frac{\epsilon^{2} Q}{P_{0} W_{1}}+O\left(\epsilon^{3}\right),
$$

which is a representation of the load-displacement curve when $\xi=\xi(\epsilon)$ in a neighborhood of $P_{0}$. Clearly, setting $\delta=0$ gives extremal values of $P(\epsilon, \delta)$. A plot of (2.22) is given in Fig. 2. This can be related to the load-displacement curve in the original variables by (2.19) and (2.5).

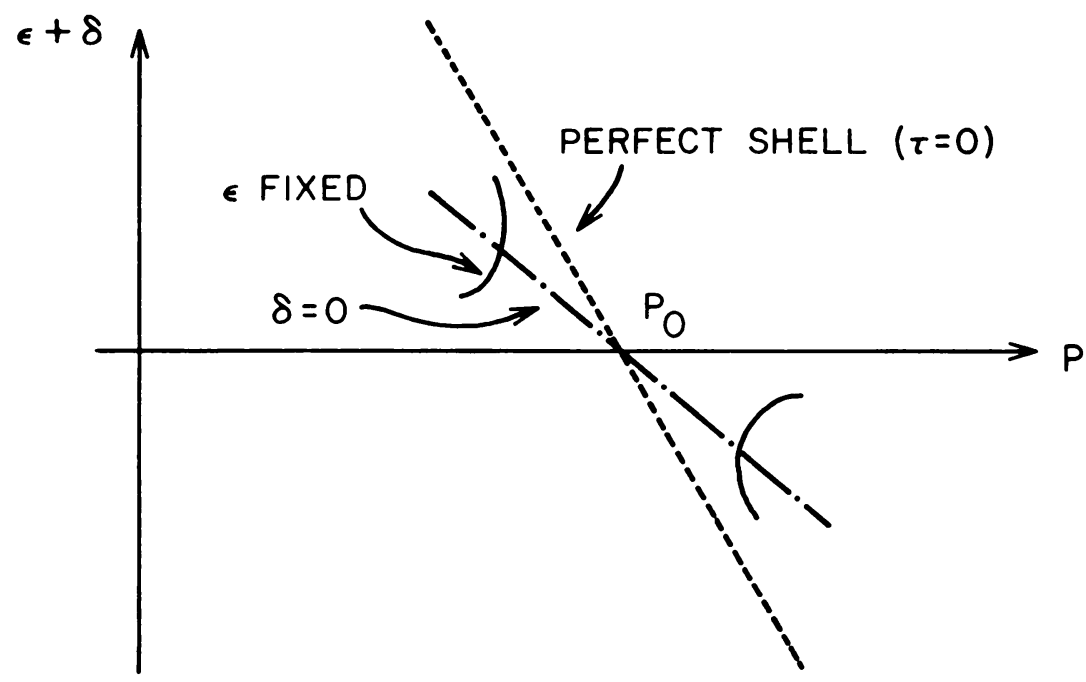

FIG. 2. Plot of load-displacement curve for spherical shell given by (2.22). 
One reason for studying this problem was to study the buckling of a clamped spherical cap. The solution (2.13) is valid only for small $\epsilon$ and hence for small $\xi$. However, one might try to find a valid extension of (2.16) so that we may allow $\xi \alpha_{1}=1$. Our method gives results pertinent to this problem when $\xi(\epsilon)$ in $(2.13)$ is positive. Examination of Table I shows that this is the case for some values of the physical parameter $\rho$ and not for others.

Now that we have determined the solution of (2.1), (2.3) with boundary condition (2.4a) it is a simple matter to find the corresponding solutions for any other boundary condition. In order to treat (2.1), (2.3) with (2.4b) we set $\tau=\xi$ in (2.11) and set $\alpha_{1}=0$, $\alpha_{1}=1$. Then we apply the transformation (2.5) to find Eq. (2.6) with the new boundary condition (2.4b). Apparently, the possible bifurcation values of $P$ are determined by the eigenvalue problem (2.7) with $\xi=v(x)=w(x)=0$ and (2.4b) replacing $v^{\prime}(1)-$ $\nu v(1)=0$. The resulting eigenvalues and eigenfunctions are

$$
\begin{aligned}
& P_{0_{n}}=\left({\lambda_{n}}^{2} / \rho\right)+\left(2 \rho / \lambda_{n}{ }^{2}\right), \\
&\left(\begin{array}{l}
\varphi_{0} \\
\psi_{0}
\end{array}\right)_{n^{\prime}}=\left(\begin{array}{c}
J_{1}\left(\lambda_{n} x\right) \\
\frac{2 \rho}{\lambda_{n}^{2}} J_{1}\left(\lambda_{n} x\right)
\end{array}\right), \\
&\left(\begin{array}{l}
\varphi_{0}^{*} \\
\psi_{0}^{*}
\end{array}\right]_{n}=\left(\begin{array}{c}
J_{1}\left(\lambda_{n} x\right) \\
\frac{-\rho}{\lambda_{n}} J_{1}\left(\lambda_{n} x\right)
\end{array}\right) .
\end{aligned}
$$

Here $\lambda_{n}=j_{1 n}$ is the $n$th zero of the Bessel function $J_{1}(x)$, and $\left(\begin{array}{c}\varphi_{0} 0^{*} \\ \psi_{0}^{*}\end{array}\right)_{n}$ is the solution of the corresponding adjoint equation. We let $P_{0}$ be that value of $P_{0_{n}}$ which is minimized over $\lambda_{n}$. With the generalized Green's function corresponding to boundary condition (2.4b) the results (2.13)-(2.22) are valid when the eigenfunctions (2.23) are appropriately substituted for the eigenfunctions (2.8). The generalized Green's function is given in the Appendix. In Table II we present some values of $P_{0}, Q$ and $P_{1}$ for various values of $\rho$, which were determined with Young's modulus chosen as $\nu=0.3$ by numerical evaluation of the integrals given in $(2.15 \mathrm{~b})$.

TABLE II

Values of the coefficients of (2.15) for the boundary-value problem $(2.1),(2.3)$ and $(2.4 b) . P_{3}=\left(P_{0} / Q\right)^{1 / 2}$.

\begin{tabular}{rrrrr}
\hline$\sqrt{ } \rho$ & $P_{0}$ & $P_{1}$ & $Q$ & \multicolumn{1}{c}{$P_{3}$} \\
\hline 1.0 & 14.82 & 0.207 & 0.0167 & 6.15 \\
2.0 & 4.21 & 0.827 & 0.067 & 6.56 \\
3.0 & 2.86 & 1.86 & 0.151 & 8.10 \\
4.0 & 3.10 & 3.31 & 0.268 & 11.24 \\
5.0 & 2.98 & 1.25 & 0.056 & 9.09 \\
6.0 & 2.83 & 1.79 & 0.081 & 10.62 \\
7.0 & 2.99 & 2.44 & 0.110 & 12.75 \\
8.0 & 2.85 & 1.67 & 0.052 & 12.49 \\
9.0 & 2.84 & 2.12 & 0.066 & 13.89 \\
10.0 & 2.90 & 1.43 & 0.034 & 13.19 \\
\hline
\end{tabular}


Appendix. The Green's functions used in Sec. II are the solutions of the equation

$$
\left(\begin{array}{ll}
L & 0 \\
0 & L
\end{array}\right) G+x\left(\begin{array}{rr}
\lambda^{2}+\frac{2 \rho^{2}}{\lambda^{2}} & -\rho \\
2 \rho & 0
\end{array}\right) G=\delta(x-\xi) I-A x\left(\begin{array}{l}
\varphi_{0}(x) \\
\psi_{0}(x)
\end{array}\right)\left(\begin{array}{l}
\varphi_{0}^{*}(\xi) \\
\psi_{0}^{*}(\xi)
\end{array}\right)^{T}
$$

with appropriate boundary conditions as given in (2.3) and (2.4). Straightforward calculation shows that with boundary conditions (2.4a), we have

$$
G(x, \xi)=\left(\begin{array}{ll}
G_{11}(x, \xi) & G_{12}(x, \xi) \\
G_{21}(x, \xi) & G_{22}(x, \xi)
\end{array}\right)
$$

where

$$
\begin{aligned}
& G_{11}(x, \xi)=\alpha \varphi_{0}(x)+\left\{\begin{array}{r}
-C_{1}(\xi) \frac{J_{1}(\lambda x)}{J_{1}(\lambda)}+\frac{\pi \xi \lambda^{2}}{2\left(\lambda^{4}-2 \rho^{2}\right)} \Theta(x, \xi)+\frac{A}{2} \varphi_{0}{ }^{*}(\xi) h_{1}(x), \\
0 \leq x<\xi \leq 1, \\
-C_{1}(\xi) \frac{J_{1}(\lambda x)}{J_{1}(\lambda)}+\frac{\pi \xi \lambda^{2}}{2\left(\lambda^{4}-2 \rho^{2}\right)} \Theta(\xi, x)+\frac{A}{2} \varphi_{0}{ }^{*}(\xi) h_{1}(x), \\
0 \leq \xi<x \leq 1,
\end{array}\right. \\
& G_{21}(x, \xi)=\alpha \psi_{0}(x)+\left\{\begin{array}{lc}
\frac{-2 \rho}{\lambda^{2}} C_{1}(\xi) \frac{J_{1}(\lambda x)}{J_{1}(\lambda)}+\frac{\pi \xi \rho \lambda^{2}}{\left(\lambda^{4}-2 \rho^{2}\right)} \Phi(x, \xi) & +A \rho \varphi_{0}^{*}(\xi) h_{2}(x), \\
\frac{-2 \rho}{\lambda^{2}} C_{1}(\xi) \frac{J_{1}(\lambda x)}{J_{1}(\lambda)}+\frac{\pi \xi \rho \lambda^{2}}{\lambda^{4}-2 \rho^{2}} \Phi(\xi, x) & 0<\xi \leq 1, \varphi_{0}^{*}(\xi) h_{2}(x), \\
& 0 \leq \xi<x \leq 1,
\end{array}\right. \\
& G_{12}(x, \xi)=\beta \varphi_{0}(x)+\left\{\begin{array}{r}
-C_{2}(\xi) \frac{J_{1}(\lambda x)}{J_{1}(\lambda)}-\frac{\pi \xi \rho \lambda^{2}}{2\left(\lambda^{4}-2 \rho^{2}\right)} \Phi(x, \xi)+A \frac{\psi_{0}^{*}(\xi)}{2} h_{1}(x), \\
0 \leq x<\xi \leq 1, \\
-C_{2}(\xi) \frac{J_{1}(\lambda x)}{J_{1}(\lambda)}-\frac{\pi \xi \rho \lambda^{2}}{2\left(\lambda^{4}-2 \rho^{2}\right)} \Phi(\xi, x)+A \frac{\psi_{0}^{*}(\xi)}{2} h_{1}(x), \\
0 \leq \xi<x \leq 1,
\end{array}\right. \\
& G_{22}(x, \xi)=\beta \psi_{0}(x)+\left\{\begin{aligned}
& \frac{-2 \rho}{\lambda^{2}} C_{2}(\xi) \frac{J_{1}(\lambda x)}{J_{1}(\lambda)}+\frac{\pi \xi \lambda^{2}}{2\left(\lambda^{4}-2 \rho^{2}\right)} \Theta\left(\frac{\sqrt{ } 2 \rho}{\lambda^{2}} x, \frac{\sqrt{ } 2 \rho}{\lambda^{2}} \xi\right) \\
&+A \rho \psi_{0}^{*}(\xi) h_{2}(x), \quad 0 \leq x<\xi \leq 1, \\
& \frac{-2 \rho}{\lambda^{2}} C_{2}(\xi) \frac{J_{1}(\lambda x)}{J_{1}(\lambda)}+\frac{\pi \xi \lambda^{2}}{2\left(\lambda^{4}-2 \rho^{2}\right)} \Theta\left(\frac{\sqrt{ } 2 \rho}{\lambda^{2}} \xi, \frac{\sqrt{ } 2 \rho}{\lambda^{2}} x\right) \\
&+A \rho \psi_{0}^{*}(\xi) h_{2}(x), \quad 0 \leq \xi<x \leq 1,
\end{aligned}\right.
\end{aligned}
$$

where 


$$
\begin{aligned}
h_{1}(x) & =x \frac{J_{1}(\mu) J_{0}(\lambda x)}{\lambda}-x \frac{J_{1}(\lambda) J_{0}(\mu x)}{\mu}, \quad \mu=\sqrt{ } 2 \rho / \lambda \\
h_{2}(x) & =x \frac{J_{1}(\mu) J_{0}(\lambda x)}{\lambda^{3}}-x \frac{J_{1}(\lambda) J_{0}(\mu x)}{\mu^{3}}, \\
\Phi(x, \xi) & =Y_{1}(\lambda \xi) J_{1}(\lambda x)-Y_{1}(\mu \xi) J_{1}(\mu x), \\
\Theta(x, \xi) & =\lambda^{2} Y_{1}(\lambda \xi) J_{1}(\lambda x)-\mu^{2} Y_{1}(\mu \xi) J_{1}(\mu x), \\
C_{1}(\xi) & =\frac{\pi \xi \lambda^{2}}{2\left(\lambda^{4}-2 \rho^{2}\right)} \Theta(1, \xi)+\frac{A}{2} \varphi_{0}^{*}(\xi) h_{1}(1), \\
C_{2}(\xi) & =\frac{-\pi \xi \rho \lambda^{2}}{2\left(\lambda^{4}-2 \rho^{2}\right)} \Phi(1, \xi)+A \frac{\psi_{0}^{*}(\xi)}{2} h_{1}(1), \\
\frac{1}{A} & =\int_{0}^{1} x\left(\varphi_{0}(x) \varphi_{0}^{*}(x)+\psi_{0}(x) \psi_{0}^{*}(x)\right) d x,
\end{aligned}
$$

and the constants $\alpha$ and $\beta$ are chosen so that

$$
\begin{aligned}
& \int_{0}^{1} x\left(\varphi_{0}^{*}(x) G_{11}(x, \xi)+\psi_{0}^{*}(x) G_{21}(x, \xi)\right) d x=0, \\
& \int_{0}^{1} x\left(\varphi_{0}^{*}(x) G_{12}(x, \xi)+\psi_{0}^{*}(x) G_{22}(x, \xi)\right) d x=0,
\end{aligned}
$$

respectively. The eigenfunctions $\varphi_{0}(x), \psi_{0}(x)$ and $\varphi_{0}{ }^{*}(x), \psi_{0}{ }^{*}(x)$ are found in (2.8).

The Green's function associated with the boundary condition (2.4b) is easier to calculate, since a simple transformation diagonalizes the system (A.1). In this case

$$
G(x, \xi)=\frac{\lambda^{2} \rho}{2 \rho^{2}-\lambda^{4}}\left(\begin{array}{cc}
1 & 1 \\
\frac{2 \rho}{\lambda^{2}} & \frac{\lambda^{2}}{\rho}
\end{array}\right)\left(\begin{array}{cc}
G_{1}(x, \xi) & 0 \\
0 & G_{2}(x, \xi)
\end{array}\right)\left(\begin{array}{cc}
\frac{-\lambda^{2}}{\rho} & 1 \\
\frac{2 \rho}{\lambda^{2}} & -1
\end{array}\right)
$$

where

$$
\begin{aligned}
& G_{1}(x, \xi)=\frac{\pi}{2} Y_{1}(\lambda \xi) J_{1}(\lambda x)-\left(\frac{2}{\lambda^{2} J_{0}^{2}(\lambda)}+\frac{\pi}{2} \frac{Y_{0}(\lambda)}{J_{0}(\lambda)}\right) J_{1}(\lambda \xi) J_{1}(\lambda x) \\
& +\frac{\xi J_{0}(\lambda \xi)}{\lambda J_{0}^{2}(\lambda)} J_{1}(\lambda x)+\frac{J_{1}(\lambda \xi)}{\lambda J_{0}^{2}(\lambda)} x J_{0}(\lambda x), \quad 0 \leq x<\xi \leq 1, \\
& =\frac{\pi}{2} J_{1}(\lambda \xi) Y_{1}(\lambda x)-\left(\frac{2}{\lambda^{2} J_{0}{ }^{2}(\lambda)}+\frac{\pi}{2} \frac{Y_{0}(\lambda)}{J_{0}(\lambda)}\right) J_{1}(\lambda \xi) J_{1}(\lambda x) \\
& +\frac{\xi J_{0}(\lambda \xi) J_{1}(\lambda x)}{\lambda^{2} J_{0}^{2}(\lambda)}+\frac{J_{1}(\lambda \xi)}{\lambda J_{0}^{2}(\lambda)} x J_{0}(\lambda x), \quad 0 \leq \xi<x \leq 1, \\
& G_{2}(x, \xi)=\frac{\pi}{2}\left(J_{1}(\mu) Y_{1}(\mu \xi)-J_{1}(\mu \xi) Y_{1}(\mu)\right) \frac{J_{1}(\mu x)}{J_{1}(\mu)}, \quad 0 \leq x<\xi \leq 1, \\
& =\frac{\pi}{2} \frac{J_{1}(\mu \xi)}{J_{1}(\mu)}\left(J_{1}(\mu) Y_{1}(\mu x)-Y_{1}(\mu) J_{1}(\mu x)\right), \quad 0 \leq \xi<x \leq 1,
\end{aligned}
$$


where $\mu=\sqrt{ } 2 \rho / \lambda$ and

$$
\int_{0}^{1} x\left(\begin{array}{l}
\varphi_{0}^{*}(x) \\
\psi_{0}^{*}(x)
\end{array}\right)^{T} G(x, \xi) d x=0
$$

\section{REFERENCES}

[1] J. C. Amazigo, Buckling of stochastically imperfect columns on nonlinear elastic foundations, Quart. Appl. Math. 29, 403-409 (1971)

[2] J. C. Amazigo, B. Budiansky and G. F. Carrier, Asymptotic analyses of the buckling of imperfect columns on nonlinear elastic foundations, Int. J. Solids Structures 6, 1341-1356 (1970)

[3] B. Budiansky, Buckling of clamped shallow spherical shells, in Symp. on theory of thin elastic shells, Delft, 1959, pp. 64-94

[4] B. Budiansky and J. W. Hutchinson, A survey of some buckling problems, AIAA J. 4, 1505-1510 (1966)

[5] R. Courant and D. Hilbert, Methods of mathematical physics, Vol. 1, Interscience, New York, 1966

[6] J. W. Hutchinson and W. T. Koiter, Post-buckling theory, Appl. Mech. Rev., 23, 1353-1366 (1970)

[7] J. W. Hutchinson, Imperfection sensitivity of externally pressurized spherical shells, J. Appl. Mech. 34, 49-55. (1967)

[8] J. P. Keener, Some modified bifurcation problems with application to imperfection sensitivity in buckling, $\mathrm{Ph}$. D. thesis, Caltech, Pasadena, 1972

[9] J. P. Keener and H. B. Keller, Perturbed bifurcation theory, Arch. Rat. Mech. Anal. 50, 159-175 (1973)

[10] - Perturbed bifurcation theory and buckling of circular plates, in Conference on ordinary and partial differential equations, University of Dundee, Springer, 1972

[11] — Multiple positive solutions of convex nonlinear eigenvalue problems (to appear in J. Diff. Eqs.)

[12] H. B. Keller and W. F. Langford, Iterations, perturbations and multiplicities for nonlinear bifurcation problems, Arch. Rat. Mech. Anal. 43, 83-103 (1972)

[13] E. L. Reiss, Bifurcation buckling of spherical caps, Comm. Pure. Appl. Mech. 18, 65-82 (1965) 